\title{
Consumption of Baits Containing Raccoon Pox-Based Plague Vaccines Protects Black-Tailed Prairie Dogs (Cynomys ludovicianus)
}

\author{
Tonie E. Rocke, Nicola Pussini, ${ }^{1, \star}$ Susan R. Smith, Judy Williamson, ${ }^{1}$ Bradford Powell, ${ }^{1}$ and Jorge E. Osorio ${ }^{3}$
}

\begin{abstract}
Baits containing recombinant raccoon poxvirus (RCN) expressing plague antigens (fraction 1 [F1] and a truncated form of the $\mathrm{V}$ protein-V307) were offered for voluntary consumption several times over the course of several months to a group of 16 black-tailed prairie dogs (Cynomys ludovicianus). For comparison, another group of prairie dogs ( $n=12$ ) was injected subcutaneously (SC) (prime and boost) with $40 \mu \mathrm{g}$ of F1-V fusion protein absorbed to alum, a vaccine-adjuvant combination demonstrated to elicit immunity to plague in mice and other mammals. Control animals received baits containing $\mathrm{RCN}$ without the inserted antigen $(n=8)$ or injected diluent $(n=7)$, and as there was no difference in their survival rates by Kaplan-Meier analysis, all of them were combined into one group in the final analysis. Mean antibody titers to Yersinia pestis $\mathrm{F} 1$ and $\mathrm{V}$ antigen increased $(p<0.05)$ in the vaccinated groups compared to controls, but titers were significantly higher $(p<0.0001)$ in those receiving injections of F1-V fusion protein than in those orally vaccinated with RCN-based vaccine. Interestingly, upon challenge with approximately $70,000 \mathrm{cfu}$ of virulent $Y$. pestis, oral vaccination resulted in survival rates that were significantly higher $(p=0.025)$ than the group vaccinated by injection with F1-V fusion protein and substantially higher $(p<0.0001)$ than the control group. These results demonstrate that oral vaccination of prairie dogs using RCN-based plague vaccines provides significant protection against challenge at dosages that simulate simultaneous delivery of the plague bacterium by numerous flea bites.
\end{abstract}

Key Words: Black-tailed prairie dogs—Cynomys ludovicianus-F1-V fusion protein-Oral vaccination-PlagueRaccoon poxvirus vaccines-Yersinia pestis.

\section{Introduction}

$\mathbf{P}$ RAIRIE dogs (CYNOMYS SPP.) ARE highly susceptible to sylvatic plague, caused by Yersinia pestis, and this zoonotic disease often decimates entire colonies of prairie dogs, causing local extinctions or regional reductions of their populations (Cully et al. 1997, Stapp et al. 2004, Pauli et al. 2006). Along with other wild rodents, prairie dogs are considered amplifying hosts for plague and a source of the disease for other wildlife, domestic animals, and humans in the western United States (Barnes 1993). Prevention of plague in wild rodents by vaccination could reduce outbreaks of the disease. However, practicable large-scale vaccination of free-ranging wildlife populations can only be achieved through voluntary consumption of vaccine. With some exceptions, most of the plague vaccines formulated to date represent poor candidates for oral vaccination, as they cannot withstand the alimentary tract or do not illicit a strong mucosal immune response (e.g., Thomas et al. 1992). Oral vaccination against plague using a live avirulent Yersinia pseudotuberculosis strain (Blisnick et al. 2008) or Salmonella-vectored vaccines expressing plague antigens (Yang et al. 2007) has been shown to protect laboratory mice, although the stability and feasibility of these products in baits used to deliver vaccines to wildlife has never been demonstrated.

Poxviruses are good candidates for the development of wildlife vaccines because of their ability to infect mucosal tissue and their stability in baits distributed in the

\footnotetext{
${ }^{1}$ USGS National Wildlife Health Center, Madison, Wisconsin.

${ }^{2}$ Bacteriology Division, U.S. Army Medical Research Institute of Infectious Diseases, Frederick, Maryland.

${ }^{3}$ School of Veterinary Medicine, University of Wisconsin, Madison, Wisconsin.

*Present address: The Marine Mammal Center Sausalito, California.
} 
environment. In previous experiments (Mencher et al. 2004, Rocke et al. 2008b), we demonstrated that recombinant raccoon poxvirus ( $\mathrm{RCN}$ ) was an effective oral delivery system for the fraction 1 (F1) capsular antigen of $Y$. pestis. Approximately $50 \%$ of black-tailed prairie dogs that voluntarily consumed oral baits with RCN-F1 survived subsequent challenge with $Y$. pestis at dosages of 70,000-130,000 $\mathrm{cfu}$, which probably represents numerous and repeated flea bites (Lorange et al. 2005).

To improve vaccine efficacy for field use, we recently constructed and optimized an additional RCN-vectored vaccine containing a truncated form of the $\operatorname{LcrV}(V)$ gene, designated herein as V307 (Rocke et al., unpublished data). Addition of the $V$ gene could also provide protection against F1-negative strains of $Y$. pestis, which have been detected in laboratory studies (Williams et al. 1978) and might occur in nature. When both vaccine constructs (RCN-F1 and RCNV307) were used to vaccinate mice in a previous study, survival upon plague challenge was significantly enhanced over animals vaccinated with either construct alone (Rocke, unpublished data). In the present study, we demonstrate that consumption of baits containing both vaccine constructs, RCN-F1 and RCN-V307, by prairie dogs resulted in nearcomplete protection to subsequent plague challenge. Their survival was better than that of prairie dogs injected SC with F1-V fusion protein, a vaccine demonstrated to induce immunity to plague in mice and other mammals (Powell et al. 2005), including black-footed ferrets (Rocke et al. 2008a).

\section{Materials and Methods}

\section{Experimental animals}

Adult black-tailed prairie dogs (males and females) were captured from wild colonies near Wall, SD $\left(43.992^{\circ} \mathrm{N}\right.$, 102.241 ${ }^{\circ} \mathrm{W}$ ), and then transported to the U.S. Geological Survey National Wildlife Health Center (Madison, WI). At the time of capture, plague was not considered endemic in this region. The animals were dusted with carbaryl before shipment, and, upon arrival at National Wildlife Health Center, they were inspected for external parasites (none were found), injected with an anthelminthic $(200 \mu \mathrm{g} / \mathrm{kg}$ of Ivermectin; Merck \& Co., West Point, PA), and then treated with $200 \mu \mathrm{L}$ of Advantage flea control (Imidacloprid; Bayer HealthCare, Shawnee Mission, KS) via external application to the skin on the back of the neck. Electronic microchip identification units (Avid Identification Systems, Folsom, LA) were inserted SC into each animal between the scapula. Prairie dogs were group-housed in isolation rooms with approximately $180 \mathrm{ft}^{2}$ of floor space. Beta chips (Northwestern Productions Corporation, Warrensburg, NY) covered the floor, and for shelter, custom-made stainless steel nest boxes with connecting lengths of polyvinyl chloride pipes were provided. An alfalfabased pelleted food (approximately $50 \mathrm{~g}$ per animal per day) and fresh vegetables (broccoli, carrot, green beans, and sweet potato chunks) were provided once daily. Water was available ad libitum.

\section{Administration of oral RCN-vectored vaccines}

Two raccoon pox-vectored recombinant plague vaccine constructs were used in this study. The first, RCN-IRES-tPAYpF1 (designated as RCN-F1 in this paper), was produced as previously described (Osorio et al. 2003) and stored at $-70^{\circ} \mathrm{C}$ in $2 \mathrm{~mL}$ aliquots until bait production. The second construct, RCN-IRES-tPA-YpV307 (designated as RCN-V307), was prepared using the $V$ gene truncated at amino acid position 307 (Rocke et al., submitted for publication). Briefly, each vaccine construct was created by replacing the thymidine kinase (TK) gene of RCN with the gene for Y. pestis F1 or V307 and associated regulatory elements (a poxvirus promoter, an internal ribosome entry site, and the secretory leader from tissue plasminogen activator). Virus stocks were thawed and diluted to $5 \times 10^{7} \mathrm{TCID}_{50} / \mathrm{mL}$ in Hank's medium (Gibco BRL, Carlsbad, CA) supplemented with 5\% glycerin (Sigma, St. Louis, MO) immediately before adding to sweet potato gelatin baits. Preparation of baits and validation of vaccine titer was described in Mencher et al. (2004). Briefly, $10 \mathrm{~g}$ shredded organic yams were mixed with $10 \mathrm{~mL}$ of Hank's medium with glycerin and $6.2 \%$ gelatin $(\mathrm{w} / \mathrm{v})$, and after the vaccine was gently mixed in, the baits were refrigerated to harden. Placebo baits were prepared similarly but contained RCN vector with the TK gene deleted $\left(\mathrm{RCN}^{-T K}{ }^{-}\right)$but no inserted genes.

Prairie dogs were prepared for bait administration by withholding fresh vegetables for $48 \mathrm{~h}$ and food pellets for $12-18 \mathrm{~h}$. All animals were then identified by microchips and individually placed in pet carriers with a small food dish containing either one vaccine-laden bait or one placebo bait, according to the experimental group. After $2-4 \mathrm{~h}$, all animals were released, and bait consumption was recorded for each individual. This process was performed again the next day to ensure bait consumption. Baits containing both RCN-F1 and RCN-V307 mixed together were offered to prairie dogs $(n=16)$ at 30-day intervals (days 1-2 and 29-30). Upon back titration of prepared baits and stocks, it was determined that the titer of the RCN-F1 construct included in the baits was too low $\left(\sim 10^{5} \mathrm{pfu}\right)$ to induce an effective immune response (based on previous experience; Rocke, unpublished data), so a new lot was prepared, and baits containing only RCN-F1 were offered to prairie dogs on days 122 and 143 . For the final bait administration on day 172, both RCN-F1 and RCN-V307 constructs were mixed together in one bait and offered to the prairie dogs. In all, prairie dogs consumed baits with approximately $10^{7} \mathrm{pfu}$ of each vaccine construct three times. Placebo baits were given to a separate group of prairie dogs $(n=8)$ at each interval.

\section{Administration of F1-V fusion vaccine}

To compare the performance of our orally delivered vaccine with a well-studied injectable protein vaccine based on the same plague antigens (F1 and V), we vaccinated a separate group of prairie dogs $(n=12)$ with F1-V fusion protein provided by the U.S. Army Medical Research Institute of Infectious Diseases (USAMRIID). This F1-V fusion protein and our methods of preparing the vaccine-adjuvant mixture for SC injection were described previously (Rocke et al. 2004, Powell et al. 2005). Briefly, the antigen was diluted in Iscove's Modified Dulbecco's medium (Sigma), mixed 1:1 with $0.2 \%$ Alhydrogel (United Vaccines, Middleton, WI), and rocked gently overnight at $4{ }^{\circ} \mathrm{C}$. Prairie dogs were vaccinated with $40 \mu \mathrm{g}$ of the diluted F1-V fusion protein mixed with adjuvant $(0.5 \mathrm{~mL}$ volume) via SC inoculation. Control animals $(n=7)$ received a placebo of $0.5 \mathrm{~mL}$ of the adjuvant (without 
antigen) suspended in Iscove's Modified Dulbecco's medium. Each group received a booster injection of protein or diluent (same dose and volume) 30 days later.

\section{Y. pestis challenge}

Three weeks after consumption of the final vaccine-laden bait and 10 weeks after the injectable vaccine administration, animals were challenged with the CO92 wild-type isolate of $Y$. pestis (provided by USAMRIID), a highly virulent and wellcharacterized strain. Stock aliquots of the bacteria, prepared and quantified as previously described (Osorio et al. 2003), were diluted 1000-fold in sterile saline and shown to have a mouse intradermal (ID) $\mathrm{LD}_{50}$ of $20 \mathrm{cfu}$. A volume of $0.2 \mathrm{~mL}$ of this solution was administered to each prairie dog by SC injection in the right hip region. Plate counts of the challenge inoculum verified that a dose of approximately 70,000 cfu (3500 mouse ID $\mathrm{LD}_{50}$ ) was given to each prairie dog, and concurrent mouse tests confirmed its virulence. Prairie dogs were monitored 2-3 times daily for 28 days for signs of illness or death. Carcasses and sick animals were removed immediately upon discovery. Animals with obvious clinical signs (labored breathing and disinclination to move) were humanely euthanized as were all survivors at the end of the 28-day period. Carcasses were frozen for future necropsy.

Upon necropsy, tissue samples from lung, liver, spleen, and skin or any visible abscess at the site of inoculation were cultured in brain heart infusion broth and on blood agar plates (Becton-Dickinson, Franklin Lakes, NJ) at $28^{\circ} \mathrm{C}$ for up to $72 \mathrm{~h}$. Plague-induced death was verified by isolating Y. pestisspecific DNA sequences from bacterial colonies using polymerase chain reaction and primers specific for the $Y$. pestis F1 gene (Heath et al. 1998). DNA fragments were fractionated and directly observed using standard techniques.

\section{Serology}

Blood samples $(\leq 300 \mu \mathrm{L})$ were collected from the medial saphenous vein of each prairie dog 1-2 days before the priming vaccination and 1-2 days before challenge; blood samples were also obtained from survivors postchallenge. Serum was collected and stored at $-20^{\circ} \mathrm{C}$ until analyses.

Antibody titers to $Y$. pestis F1 antigen were determined using enzyme-linked immunosorbent assay with F1 antigen and V antigens supplied by USAMRIID, as described previously (Rocke et al. 2008b). Briefly, serum samples were serially diluted fourfold from 1:160 to 1:40,960; test samples were run in duplicate. Each plate also contained four replicates of a negative control serum sample and two replicates of a positive control serum sample. A horseradish peroxidase-labeled anti-prairie dog IgG custom prepared by Bethyl Laboratories (Montgomery, TX) was diluted 1:100 and used as the secondary antibody. Titers $<1: 160$ were considered negative and recorded as 1:40. The highest dilution that was positive (exceeded the mean of four negative control samples by three standard deviations) was considered the endpoint and its reciprocal value recorded as the titer.

\section{Statistical analyses}

All statistical analyses were performed using SAS software (SAS 2005). The Kaplan-Meier survival analysis was used to calculate survival curves, followed by log-rank tests to de- termine significance. Because Kaplan-Meier analysis showed that survival was not significantly different between the two control groups (log rank chi-square; $p=0.37$ ), they were pooled into one group for further analyses. Antibody titers were transformed by calculating the $\log _{10}$ of the titer to symmetize the data and improve the accuracy of confidence interval coverage. For comparing antibody titers between groups, we used a robust linear models approach, which involves first transforming the data to ranks and then applying the appropriate linear models tests: a $t$-test for two groups or a one-way analysis of variance for more than two groups. This approach is equivalent to the nonparametric Wilcoxon and Kruskal-Wallis tests, respectively (Conover and Iman 1981). For comparing antibody titers pre- and postvaccination or pre- and postchallenge, a matched-pairs analysis was used.

\section{Results}

The primary objective of this study was to measure antibody response and survival to challenge of prairie dogs orally vaccinated against plague with RCN-based vaccines in comparison to controls and animals that received an injectable vaccine. Prairie dogs consumed $\geq 75 \%$ of the RCN vaccineladen bait offered to them, and no adverse reactions were observed in any of the animals that received these or the injectable F1-V vaccine.

Prairie dogs that consumed RCN vaccine-laden baits had significantly higher $(p<0.05)$ mean IgG titers to F1 and V antigens prechallenge compared to their prevaccination levels and the prechallenge titers of control animals (Fig. 1), although the rise was only about 10-20-fold. In comparison, animals that received $\mathrm{F} 1-\mathrm{V}$ vaccine injections had a significantly higher rise in titer (70-100-fold) compared to their prevaccination levels and significantly higher levels $(p<$ $0.0001)$ than both oral vaccinates and controls. Interestingly,

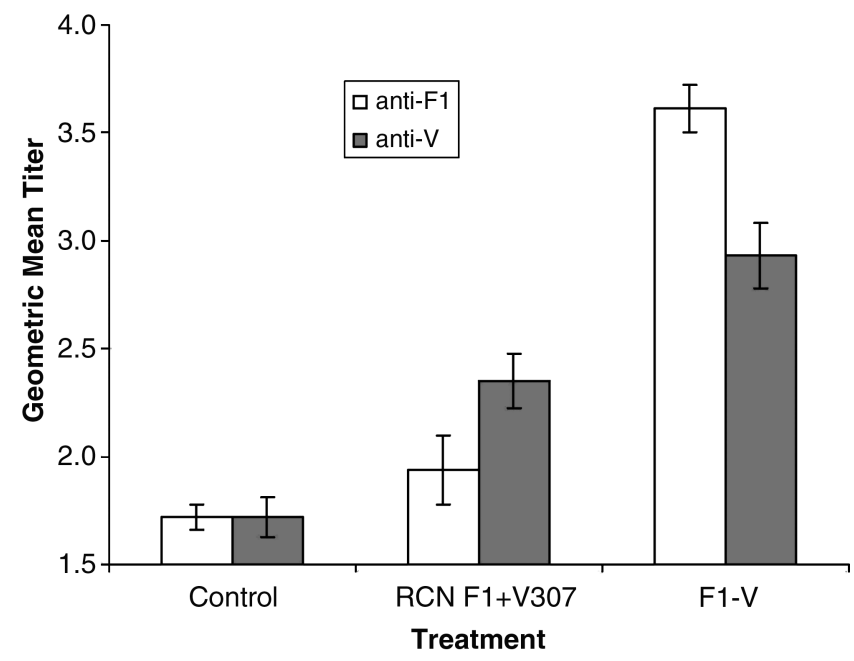

FIG. 1. Geometric mean serum immunoglobulin G antibody titers against F1 and V antigens in black-tailed prairie dogs that were vaccinated against plague by consuming RCN-F1 and RCN-V307 vaccine-laden baits or by parenteral injection of F1-V fusion protein. Mean prechallenge titers of all vaccinates were higher than controls $(p<0.05)$. Mean titers of animals injected with F1-V fusion protein were higher $(p<0.001)$ than those that consumed RCN vaccines in baits. $\mathrm{F} 1$, fraction 1 ; RCN, recombinant raccoon poxvirus. 


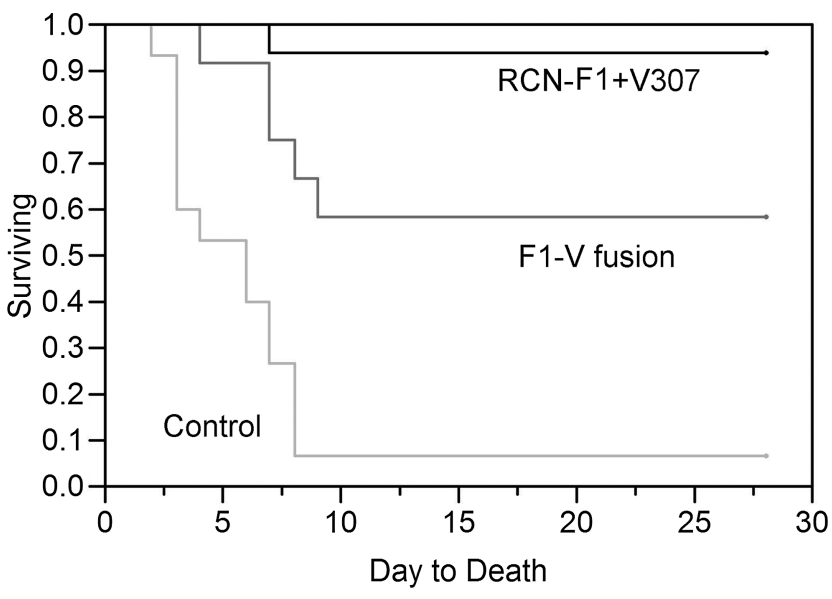

FIG. 2. Kaplan-Meier survival curves of black-tailed prairie dogs after Yersinia pestis challenge in relation to vaccine treatment. Survival of prairie dogs consuming RCN vaccineladen baits was higher than that of the group that received injections of F1-V fusion protein $(p=0.025)$ and the control group $(p<0.0001)$.

in oral vaccinates that survived challenge, $\operatorname{IgG}$ titers to $\mathrm{F} 1$ and $\mathrm{V}$ antigens increased significantly (df $14, p<0.001$ ) from a mean prechallenge titer of $1: 738$ and 1:843, respectively, to a mean postchallenge titer of $1: 16,708$ and $1: 7648$. In contrast, mean postchallenge titers of animals that received F1-V vaccine injections did not rise significantly ( $\mathrm{df} 6, p>0.05$ ) over their peak prechallenge values. Anti-F1 antibody was detected in the one surviving control animal at a very high titer $(1: 163,480)$, but no anti-V antibody was detected.

Upon challenge with $Y$. pestis, 15/16 prairie dogs that consumed vaccine-laden baits survived in comparison to $7 / 12$ prairie dogs that received F1-V vaccine injections and $1 / 15$ controls. Y.pestis was cultured from tissues of those that died, confirming that plague was the cause of death. Kaplan-Meier analysis showed that a significant difference in survival (Fig. 2) was evident between the three treatment groups $(p<0.0001)$, and that vaccinates, regardless of treatment, survived better $(p<0.0001)$ than the controls. Survival of prairie dogs consuming RCN vaccine-laden baits was higher than the group that received injections of F1-V fusion protein $(p=0.025)$. For animals that succumbed to plague, whether vaccinated or not, no overt difference $(p>0.27)$ in time to death was evident between these three groups.

\section{Discussion}

To develop an oral vaccine to protect prairie dogs against plague, we assessed the immunogenicity and protection induced from consumption of baits containing recombinant RCN vaccine vectors expressing $Y$. pestis antigens in comparison to injection of the well-characterized F1-V fusion subunit vaccine. Surprisingly, a higher level of protection against plague was achieved in prairie dogs consuming recombinant RCN vaccine-laden baits than with parenteral vaccination of F1-V fusion protein, despite the fact that the protein vaccine stimulated much higher serum IgG titers. In other studies, the F1-V fusion protein was shown to fully protect mice against $Y$. pestis challenge doses up to $9 \times 10^{6} \mathrm{LD}_{50}$
(Heath et al. 1998, Powell et al. 2005), and single-dose vaccinations even protected mice against aerosol challenge by wild-type and nonencapsulated (F1-) Y. pestis (Powell et al. 2005). However, in prairie dogs vaccinated with two injections of F1-V fusion protein in this study, only $56 \%$ of animals survived challenge with approximately 3500 mouse $\mathrm{LD}_{50}$ of $Y$. pestis. This corroborates an earlier pilot study conducted similarly in which $5 / 8$ prairie dogs vaccinated with F1-V survived challenge with the same dose of plague administered 3 weeks after receiving the booster injection (Rocke, unpublished data). In contrast, in prairie dogs orally vaccinated with recombinant RCN vaccines, nearly all (94\%) survived $Y$. pestis challenge, even though their mean IgG titers to F1 and V antigens $(<1: 1000)$ were 10 -fold lower than animals vaccinated with F1-V fusion protein. This suggests a profound difference in the role of humoral immunity in evading plague infection between mice and prairie dogs. Although not measured in this study, perhaps mucosal immunity is more critical for prairie dog survival to $Y$. pestis exposure.

Typically, the gastrointestinal tract has well-developed lymphoid tissue, enabling the stimulation of both systemic and mucosal immunity, so it is likely that other aspects of the immune system were stimulated in the orally vaccinated prairie dogs. For example, other investigators have shown that an oral plague vaccine using Salmonella-vectored F1 and $\mathrm{V}$ antigens resulted in elevations in both serum IgG and secretory IgA in laboratory mice (Yang et al. 2007), resulting in near-complete protection against a $Y$. pestis challenge dose of $1000 \mathrm{LD}_{50}$. Unfortunately, in prairie dogs, we were only able to measure serum IgG, as anti-prairie $\operatorname{dog} \operatorname{Ig} A$ is not currently available, and techniques for measuring cellular immunity are likewise limited for outbred animals such as these.

Findings herein also establish that a second, non-F1 antigen is important for inducing immune protection in prairie dogs. In two previous studies (Mencher et al. 2004, Rocke et al. 2008b), prairie dogs were orally vaccinated with baits containing only RCN-F1, and even after consuming bait three times, only about $50 \%$ survived plague challenge. In this study, prairie dogs were provided baits with RCN-V307 that expresses a truncated form of the $V$ gene, in addition to RCNF1, and nearly all survived plague challenge. Although we did not directly compare these results to the prior treatment, we believe that the significantly improved prairie dog survival was due to addition of RCN-V307. In a related study, mice simultaneously injected with RCN-F1 and RCN-V307 via the intramuscular route had higher survival rates upon plague challenge than animals that received either construct alone (Rocke et al., unpublished data). Other investigators have demonstrated that substantial improvement in subunit vaccine performance against encapsulated and nonencapsulated strains is achieved by including both F1 and V antigens, as is well-established in the mouse plague model (Anderson et al. 1996, Powell et al. 2005 and references therein). Our findings extend this knowledge to prairie dogs and highlight the critical need for a vaccine containing both antigens, because F1-negative strains of $Y$. pestis have been described and could occur in nature (Williams et al. 1978). Although we have not confirmed $\mathrm{V}$ protein-induced suppression of prairie dog immunity, we used a truncated form of the $V$ gene to avoid potential immunosuppressive properties that have been demonstrated with the full $\mathrm{V}$ protein in mice (Overheim et al. 2005). 
Our challenge dose of prairie dogs in this experiment was $7 \times 10^{4} \mathrm{cfu} Y$. pestis, a dosage that is probably equivalent to that delivered by several infectious flea bites. A recent study showed that the oriental rat flea (Xenopsylla cheopis) transmitted from 2 to 4970 cfu of $Y$. pestis per bite, an average of $636 \pm 1424$ (Lorange et al. 2005). Although similar transmission rates per flea bite are not available for prairie dog fleas (Oropsylla spp.), in a recent laboratory study by Wilder et al. (2008), median bacterial loads of $1.2-6.7 \times 10^{4} \mathrm{cfu} /$ flea were detected in O. tuberculata cynomuris $24-96 \mathrm{~h}$ after an infectious blood meal. Natural transmission of $Y$. pestis by flea-bite typically occurs via ID inoculation and leads to either bubonic plague or primary septicemic plague (Sebbane et al. 2006). In our challenge study of prairie dogs, we used SC inoculation that leads primarily to septicemic plague. Although this does not exactly replicate the pathogenesis of naturally transmitted plague, we propose that the disease outcomes would be similar enough for these results to be relevant. The $\mathrm{LD}_{50} \mathrm{~s}$ for ID- and SC-inoculated $Y$. pestis in mice are not significantly different (Rocke, unpublished data). Unfortunately, the $\mathrm{LD}_{50}$ of our challenge inoculum for prairie dogs is unknown. Several previous attempts were made to determine the $\mathrm{LD}_{50}$ for black-tailed prairie dogs (Rocke, unpublished data) before this experiment, but reproducible results in either group or singly housed animals could not be achieved. In this and our two previously published vaccine studies, 1-2 control prairie dogs $(7 \%, 11 \%$, and $12 \%)$ consistently survived challenge doses of 70,000 (Rocke et al. 2008) and as high as 130,000 cfu (Mencher at al. 2004). All of the prairie dogs in our studies were group-housed, pre- and postchallenge, to reduce the stress of isolation. Although the possibility of postchallenge cross transmission via direct contact exists, its impact in relation to the inoculation was probably minimal. In several previous challenge studies, uninfected contact control prairie dogs were housed with control groups postchallenge (a total of 10), and only 1 of these animals contracted plague (Rocke et al. 2008). Each group of animals were checked 2-3 times daily postchallenge, and any obviously sick and dead animals were removed immediately.

Current efforts to halt the spread of plague in prairie dog colonies typically rely on dusting individual prairie dog burrows with pesticides that kill plague-infected fleas after a plague outbreak has begun (Seery et al. 2003, Hoogland et al. 2004). However, pesticide application is labor intensive, costly, and difficult to sustain over time. Most important, such programs are often initiated too late to substantially affect colony survival. Our recombinant $\mathrm{RCN}$ vaccines, which provide effective immunity against plague via the oral route, could provide a practical, alternative approach, although more work is needed to determine the timing of vaccine/bait delivery, the duration of immunity provided, and the effects on nontarget animals. Potential baits that maintain vaccine stability which are also suitable for field use and could be broadcast from planes or vehicles are currently being tested. Reduction of plague outbreaks in prairie dogs through oral vaccination in targeted locations would minimize the risk of disease transfer to other animals, particularly the endangered black-footed ferret, which relies exclusively on prairie dogs for food and habitat. Such an approach would enhance prairie dog and ferret conservation while also contributing to the protection of public health.

\section{Acknowledgments}

Technical and animal care assistance was provided by J. Lambert-Newman, L. Stickney, and S. Meier. Funding for this project was provided by the USGS-Biological Resources Division and the Defense Advance Research Projects Agency. Opinions, interpretations, conclusions, and recommendations are those of the authors and are not necessarily endorsed by the U.S. Army.

\section{Disclosure Statement}

No competing financial interests exist.

\section{References}

Anderson, GW, Leary, SE, Williamson, ED, Titball, RW, et al. Recombinant $\mathrm{V}$ antigen protects mice against pneumonic and bubonic plague caused by F1-capsule-positive and -negative strains of Yersinia pestis. Infect Immun 1996; 64:4580-4585.

Barnes, AM. A review of plague and its relevance to prairie dog populations and the black-footed ferret. In: Oldemeyer, JL, Biggins, DE, Miller, BJ, eds. Proceedings of the Symposium on the Management of Prairie Dog Complexes for the Reintroduction of the Black-Footed Ferret. Washington, DC: U.S. Department of Interior Biological Report, 1993; 13:28-37.

Blisnick, T, Ave, P, Huerre, M, Carniel, E, et al. Oral vaccination against bubonic plague using a live avirulent Yersinia pseudotuberculosis strain. Infect Immun 2008; 76:3808-3816.

Conover, WJ, Iman, RI. Rank transformations as a bridge between parametric and nonparametric statistics. Am Stat 1981; 35:124-134.

Cully, JF, Jr., Barnes, AM, Quan, TJ, Maupin, G. Dynamics of plague in a Gunnison's prairie dog colony complex from New Mexico. J Wildl Dis 1997; 33:706-719.

Heath, DG, Anderson, GW, Jr., Mauro, JM. Protection against experimental bubonic and pneumonic plague by a recombinant capsular F1-V antigen fusion protein vaccine. Vaccine 1998; 16:1131-1137.

Hoogland, J, Davis, S, Benson-Amram, S, Labruna, D, et al. Pyraperm kills fleas and halts plague among Utah prairie dogs. Southwest Nat 2004; 49:376-383.

Lorange, EA, Race, BL, Sebbane, F, Hinnebusch, BJ. Poor vector competence of fleas and the evolution of hypervirulence in Yersinia pestis. J Infect Dis 2005; 191:1907-1912.

Mencher, JS, Smith, SR, Powell, TD, Stinchcomb, DT, et al. Protection of black-tailed prairie dogs (Cynomys ludovicianus) against plague after voluntary consumption of baits containing recombinant raccoon poxvirus vaccine. Infect Immun 2004; 72:5502-5505.

Osorio, JE, Powell, TD, Tomlinson, CC, Frank, RS, et al. Raccoon pox viral-vectored F1 vaccine protects mice against lethal plague. Vaccine 2003; 21:1232-1238.

Overheim, KA, DePaolo, RW, DeBord, KL, MOrrin, EM, et al. LcrV plague vaccine with altered immunomodulatory properties. Infect Immun 2005; 73:5152-5159.

Pauli, JN, Buskirk, SW, Williams, ES, Edwards, WH. A plague epizootic in the black-tailed prairie dog (Cynomys ludovicianus). J Wildl Dis 2006; 42:74-80.

Powell, B, Andrews, G, Enama, J, Jendrek, S, et al. Design and testing for a non-tagged $\mathrm{F} 1-\mathrm{V}$ fusion protein as vaccine antigen against bubonic and pneumonic plague. Biotech Prog 2005; 21:1490-1510.

Rocke, TE, Mencher, J, Smith, SR, Friedlander, AM. Recombinant F1-V fusion protein protects black-footed ferrets 
(Mustela nigripes) against virulent Yersinia pestis infection. J Zoo Wildl Med 2004; 35:142-146.

Rocke, TE, Smith, SR, Marinari, P, Kreeger, J, et al. Vaccination with F1-V fusion protein protects black-footed ferrets (Mustela nigripes) against plague upon oral challenge with Yersinia pestis. J Wildl Dis 2008a; 44:1-7.

Rocke, TE, Smith, SR, Stinchcomb, DT, Osorio, JE. Immunization of black-tailed prairie dogs against plague through consumption of vaccine-laden baits. J Wildl Dis 2008b; 44:930-937.

Sebbane, F, Jarrett, CO, Gardner, D, Long, D, et al. Role of the Yersinia pestis plasminogen activator in the incidence of distinct septicemic and bubonic forms of flea-borne plague. PNAS 2006; 3:5526-5530.

Seery, DB, Biggins, DE, Montenieri, JA, Enscore, RE, et al. Treatment of black-tailed prairie dog burrows with deltamethrin to control fleas (Insecta:Siphonaptera) and plague. J Med Entomol 2003; 40:718-722.

Stapp, P, Antolin, MF, Ball, M. Patterns of extinction in prairiedog metapopulations: plague outbreaks follow El Niño events. Fron Ecol Environ 2004; 2:235-240.

Thomas, RE, Simpson, WJ, Perry, LL, Schwan, TG. Failure of intragastrically administered Yersinia pestis capsular antigen to protect mice against challenge with virulent plague: suppression of fraction 1-specific antibody response. Am J Trop Med Hyg 1992; 47:92-97.

Wilder, AP, Eisen, RJ, Bearden, SW, Montenieri, JA. Transmission efficiency of two flea species (Oropsylla tuberculata cynomuris and Oropsylla hirsuta) involved in plague epizootics among prairie dogs. EcoHealth 2008; 5:205-212.

Williams, JE, Harrison, DN, Quan, TJ, Mullins, JL. Atypical plague bacilli isolated from rodents, fleas, and man. Am J Public Health 1978; 68:262-264.

Yang, X, Hinnebusch, BJ, Trunkle, T, Bosio, CM, et al. Oral vaccination with Salmonella simultaneously expressing Yersinia pestis $\mathrm{F} 1$ and $\mathrm{V}$ antigens protects against bubonic and pneumonic plague. J Immunol 2007; 178:1059-1067.

Address correspondence to:

Tonie E. Rocke

USGS National Wildlife Health Center 6006 Schroeder Road Madison, WI 53711

E-mail: trocke@usgs.gov 